\title{
Poly (I:C) transfection induces mitochondrial-mediated apoptosis in cervical cancer
}

\author{
HUI CHEN, DONG-LIANG WANG and YU-LING LIU \\ Department of Obstetrics and Gynecology, The Second Affiliated Hospital, \\ Zhengzhou University, Zhengzhou, Henan 450014, P.R. China
}

Received January 7, 2015; Accepted November 2, 2015

DOI: $10.3892 / \mathrm{mmr} .2016 .4848$

\begin{abstract}
Polyinosinic acid:polycytidylic acid, known as poly (I:C), is an analogue of double-stranded RNA, which exhibits direct antitumor effects against several types of cancer. The present study aimed to evaluate the role of poly (I:C) in the apoptosis of cervical cancer cells. The HeLa human cervical cancer cell line was used in the present study, and cell apoptosis was determined following poly (I:C) transfection. Furthermore, the mRNA levels of interferon (IFN)- $\beta$, the production of reactive oxygen species (ROS), DNA damage, mitochondrial membrane potential $(\Delta \Psi \mathrm{m})$ and the release of cytochrome $c$, as well as caspase activation, were determined. The effect of IFN- $\beta$ on poly (I:C) transfection-mediated apoptosis was also examined by IFN- $\beta$ knockdown. The results showed that poly (I:C) transfection markedly induced HeLa apoptosis, increased the protein levels of pro-apoptotic B cell lymphoma-2 (Bcl-2)-associated $\mathrm{X}$ protein (Bax) and $\mathrm{BH} 3$ interacting-domain death agonist (Bid), and suppressed the protein expression levels of anti-apoptotic Bcl-2 and Survivin. However, poly (I:C) transfection increased the mRNA levels of IFN- $\beta$, induced ROS production and increased the levels of phosphorylated $\gamma \mathrm{H} 2 \mathrm{~A}$.X, an indicator of DNA damage. In addition, poly (I:C) transfection decreased $\Delta \Psi \mathrm{m}$, triggered the release of cytochrome $c$ from the mitochondria to the cytosol, and induced caspase- 9 and -3 activation. IFN- $\beta$ knockdown decreased the poly (I:C)-induced production of ROS and DNA damage, restored $\Delta \Psi \mathrm{m}$ and cytochrome $c$ release, and suppressed caspase- 9 and -3 activation, thereby suppressing poly (I:C)-mediated apoptosis in the HeLa cells. Together, the results of the present study demonstrated that poly $(\mathrm{I}: \mathrm{C})$ transfection induced IFN- $\beta$, contributing to ROS production, DNA damage, and caspase- 9 and -3 activation in the HeLa cervical cancer cell line, leading to mitochondrial-mediated apoptosis.
\end{abstract}

Correspondence to: Dr Yu-Ling Liu, Department of Obstetrics and Gynecology, The Second Affiliated Hospital, Zhengzhou University, 2 Jingba Road, Zhengzhou, Henan 450014, P.R. China E-mail: yulingliusec@163.com

Key words: polyinosinic acid:polycytidylic acid, mitochondrial, apoptosis, cervical cancer

\section{Introduction}

Cervical cancer is one of the most common types of cancer among women, and is a major contributor to morbidity and mortality rates in women worldwide $(1,2)$. Inducing cancer cell apoptosis has been a critical strategy in cancer therapy, and has been the aim of several research groups. Apoptosis is triggered predominantly through the extrinsic or intrinsic caspase-dependent pathways, specifically caspase-8 and -9, respectively $(3,4)$. Mitochondria are the most important sensors for apoptosis in the intrinsic caspase-dependent pathways (5). There is also crosstalk between the extrinsic and intrinsic caspase-dependent pathways, and activation of caspase- 8 transforms BH3 interacting-domain death agonist (Bid) into truncated Bid, thereby promoting mitochondrial-mediated caspase-9-dependent apoptosis $(6,7)$. Reactive oxygen species (ROS) also induce intrinsic apoptosis by triggering DNA damage (8).

Synthetic double-stranded (ds)RNA, including polyinosinic acid:polycytidylic acid, or poly (I:C), is a mimic of viral dsRNA and is, therefore, a promising immune stimulant candidate for vaccines directed against intracellular pathogens. Previous investigation revealed that poly (I:C) suppresses the growth of murine melanoma B16F10 cells (9). Studies have also reported that combining poly (I:C) with the Toll-like receptor 9 agonist, CpG oligodeoxynucleotide (ODN), results in a more marked pro-apoptotic effect on human hepatocellular carcinoma cells, compared with using either $\mathrm{CpG}$ ODN or poly (I:C) alone (10). Poly (I:C)-containing liposome transfection promotes cell apoptosis in human hepatic carcinoma, which correlates with the upregulation of retinoic acid-inducible gene-I-like receptors (11). However, the effect of poly (I:C) on apoptosis in cervical cancer remains to be fully elucidated.

Interferons (IFNs) are multifunctional cytokines, which regulate cellular and immune responses as well as antiviral and antitumor activity (12). In addition, IFNs have been generally considered to be anti-proliferative proteins $(13,14)$. IFNs are divided into two groups: Type I IFNs and type II IFNs. Type I IFNs (IFN- $\alpha$ and IFN- $\beta$ ) markedly inhibit tumor cell growth and induce apoptosis in vitro and in vivo $(15,16)$. It has been reported that IFN- $\beta$ inhibits glioma angiogenesis through the downregulation of vascular endothelial growth factor and the upregulation of IFN-inducible protein 10 (17). Low levels of constitutively produced endogenous IFN- $\beta$ are 
sufficient to restrict tumor angiogenesis (18). Previous investigation has shown that poly $(\mathrm{I}: \mathrm{C})$ transfection induces the endogenous expression of IFN- $\beta$, which results in cell cycle arrest in human renal carcinoma cells (19). However, whether IFN- $\beta$ is involved in poly $(\mathrm{I}: \mathrm{C})$ transfection-induced apoptosis in cervical cancer remains to be elucidated.

In the present study, the effect and underlying mechanisms of poly (I:C) transfection on the HeLa human cervical cancer cell line were investigated. The present study aimed to provide evidence supporting the potential use of poly (I:C) for the treatment of cervical cancer.

\section{Materials and methods}

Cell line and cell culture. The HeLa human cervical cancer cell line was purchased from American Type Culture Collection (Manassas, VA, USA). The cells were cultured in Dulbecco's modified Eagle's medium (DMEM; Gibco; Thermo Fisher Scientific, Inc., Waltham, MA, USA) supplemented with $10 \%$ (v/v) fetal bovine serum (Gibco; Thermo Fisher Scientific, Inc.), $100 \mathrm{U} / \mathrm{ml}$ penicillin, $100 \mu \mathrm{g} / \mathrm{ml}$ streptomycin (Gibco; Thermo Fisher Scientific, Inc.) and $2.5 \mu \mathrm{g} / \mathrm{ml}$ amphotericin B (Sangon Biotech Co., Ltd., Shanghai, China) at $37^{\circ} \mathrm{C}$ in a $5 \%$ $\mathrm{CO}_{2}$ incubator. The medium was replaced every 2 days.

Poly (I:C) transfection. For poly (I:C) transfection, $1 \times 10^{5} \mathrm{HeLa}$ cells were seeded in a 12 -well plate and maintained at $37^{\circ} \mathrm{C}$ in a $5 \% \mathrm{CO}_{2}$ incubator for $15 \mathrm{~h}$. A dose of $2 \mu \mathrm{l}$ Lipofectamine ${ }^{\mathrm{TM}} 2000$ (Invitrogen; Thermo Fisher Scientific, Inc.) was added to $100 \mu \mathrm{l}$ of serum-free medium, following which the mixture was incubated at room temperature for $5 \mathrm{~min}$. At the same time, $10 \mu 1$ poly (I:C), purchased from Sigma-Aldrich (St. Louis, MO, USA) was added to the $100 \mu \mathrm{l}$ of serum-free medium. Subsequently, the Lipofectamine ${ }^{\mathrm{TM}} 2000$ and poly (I:C) were mixed gently and incubated at room temperature for $20 \mathrm{~min}$. Following incubation, the cell culture medium was replaced with serum-free medium, which was added to each well containing the Lipofectamine ${ }^{\mathrm{TM}} 2000$ and poly (I:C) mixture, and incubated at $37^{\circ} \mathrm{C}$ for $4 \mathrm{~h}$. Finally, the medium in each well was replaced with a fresh serum-containing medium.

Analysis of cell apoptosis. Cell apoptosis was measured by flow cytometry using an Annexin V-propidium iodide (PI) kit (cat. no. 556420; BD Pharmingen, San Diego, CA, USA), according to the manufacturer's protocol. Briefly, the cells were harvested and washed three times with phosphate-buffered saline (PBS). Following centrifugation at $300 \mathrm{x} g$ for $10 \mathrm{~min}$ at $4^{\circ} \mathrm{C}$, the cells were resuspended in $500 \mu \mathrm{l}$ binding buffer (0.1 M HEPES/NaOH, pH 7.4; $1.4 \mathrm{M} \mathrm{NaCl} ; 25 \mathrm{mM}$ $\mathrm{CaCl}_{2}$ ) containing $5 \mu \mathrm{l}$ fluorescein isothiocyanate-conjugated Annexin $\mathrm{V}$, the mixture was incubated at $25^{\circ} \mathrm{C}$ in the dark for $10 \mathrm{~min}$, following which $5 \mu \mathrm{l}$ PI was added. Finally, cell apoptosis was analyzed using flow cytometry (FACSCalibur; BD Biosciences, San Jose, CA, USA) with CellQuest software (BD Biosciences), with the results are expressed as a percentage of the total cells counted.

Western blot analysis. The proteins were extracted from the cells using radioimmunoprecipitation assay lysis buffer (Beyotime Institute of Biotechnology, Nantong, China).
Western blot analyses were performed, as previously reported (17). Briefly, total protein was quantified using a bicinchoninic acid kit (Beyotime Institute of Biotechnology) and $40 \mu \mathrm{g}$ protein per lane was separated by $12 \%$ sodium dodecyl sulfate-polyacrylamide gel electrophoresis prior to electroblotting onto a nitrocellulose membrane (GE Healthcare, Munich, Germany). Non-specific binding was blocked by incubating the membrane with 5\% non-fat milk in Tris-buffered-saline with Tween (TBST; $10 \mathrm{mM}$ Tris- $\mathrm{HCl}, \mathrm{pH} 7.5 ; 150 \mathrm{mM}$ $\mathrm{NaCl} ; 0.05 \%$ Tween-20) at room temperature for $1 \mathrm{~h}$. After blocking, the membrane was incubated with various primary antibodies overnight at $4{ }^{\circ} \mathrm{C}$. The antibodies used included the following: Mouse monoclonal anti-cytochrome $c(1: 1,000$; cat. no. ab13575; Abcam, Cambridge, UK), rabbit polyclonal anti-cleaved caspase-9 (1:500; cat. no. ab2325; Abcam) and -3 (1:500; cat. no. ab13847; Abcam), rabbit polyclonal anti-IFN- $\beta$ (1:400; cat. no. sc-83256; Santa Cruz Biotechnology, Inc., Dallas, TX, USA), rabbit polyclonal anti-phosphorylated (p)-H2A.X (cat. no. 07-627; 1:1,500; EMD Millipore, Billerica, MA, USA) and rabbit polyclonal anti- $\beta$-actin $(1: 2,000$; cat. no. ab59381; Abcam). The membrane was then incubated at room temperature for $2 \mathrm{~h}$ with anti-mouse horseradish peroxidase-conjugated $(1: 5,000)$ secondary antibodies (cat. no. sc-2497), obtained from Santa Cruz Biotechnology Inc. The blots were visualized by enhanced chemiluminescence (Amersham Pharmacia Biotech Inc., Piscataway, NJ, USA) and normalized to $\beta$-actin.

Reverse transcription-quantitative polymerase chain reaction $(R T-q P C R)$ analysis. The total RNA was isolated from the HeLa cells using TRIzol reagent (Invitrogen; Thermo Fisher Scientific, Inc.). Total RNA (1-2 $\mu \mathrm{g}$ ) was reverse transcribed using SuperScript ${ }^{\circledR}$ IV Reverse Transcriptase (Invitrogen; Thermo Fisher Scientific, Inc.). The RT-qPCR reactions were performed on a Rotor-Gene RG-3000 Real-Time Thermal Cycler (Corbett Research, Sydney, Australia) using a SYBR ${ }^{\circledR}$ Premix Ex Taq ${ }^{\mathrm{TM}}$ II kit (Takara Biotechnology Co., Ltd., Dalian, China). PCR primers specific for IFN- $\beta$ were designed, as previously reported (20): sense 5'-TTGAATGGG AGGCTTGAATA-3' and antisense 5'-CTATGGTCCAGG CACAGTGA-3'. These primers were synthesized by Takara Biotechnology Co., Ltd. The PCR procedure was as follows: Polymerase activation for $30 \mathrm{sec}$ at $95^{\circ} \mathrm{C}, 40$ cycles of amplification, each consisting of $95^{\circ} \mathrm{C}$ for $5 \mathrm{sec}$ and $60^{\circ} \mathrm{C}$ for $20 \mathrm{sec}$, and 1 cycle of dissociation consisting of $95^{\circ} \mathrm{C}$ for $15 \mathrm{sec}, 60^{\circ} \mathrm{C}$ for $30 \mathrm{sec}$ and $95^{\circ} \mathrm{C}$ for $15 \mathrm{sec}$. All reactions were performed in triplicate. Fluorescence data were analyzed using Rotor-Gene 6 software (version 6.0; Corbett Research). The mRNA expression levels were calculated using the $2^{-\Delta \Delta \mathrm{Cq}}$ method (21), and were normalized to $\beta$-actin and reported as arbitrary units.

Measurement of ROS. The generation of intracellular ROS generation in the HeLa cells was evaluated in the homogenate using 5-(and-6)-carboxy-2', 7'-dichlorohydrofluorescein diacetate (carboxy-H2DCFDA; DCFH), which is a specific ROS-detecting fluorescent dye. DCFH is sensitive to ROS, and can be oxidized to the highly fluorescent dichlorofluorescein (DCF) (22). The protocol was performed, according to a previous report (23). Briefly, $1 \times 10^{6} \mathrm{HeLa}$ cells were incubated with $10 \mu \mathrm{l} \mathrm{DCFH}$ (Sigma-Aldrich) for $30 \mathrm{~min}$ at $37^{\circ} \mathrm{C}$ in the 
A

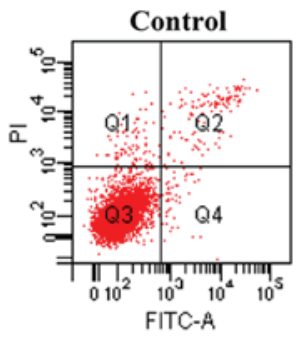

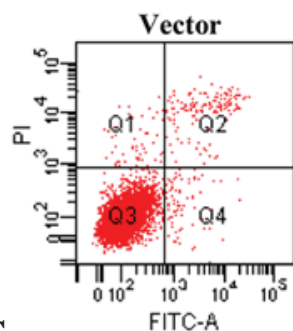

C

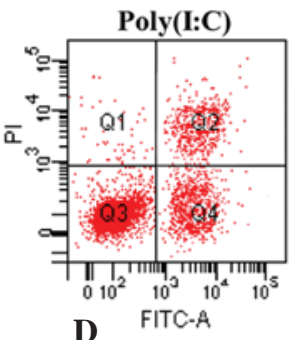

D
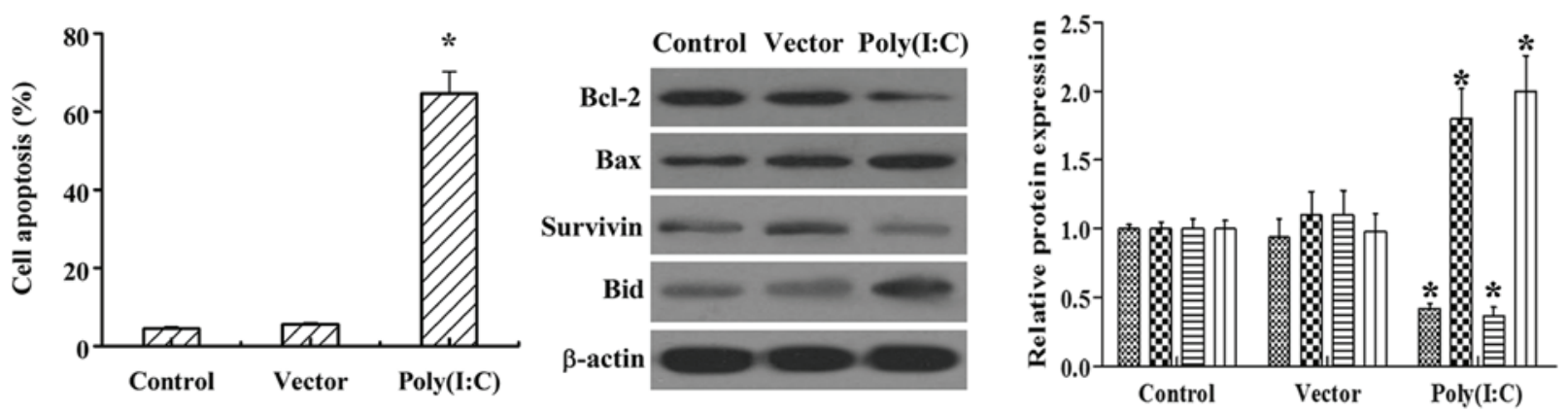

网 Bcl-2

B Bax

曰 Survivin

m Bid

Figure 1. Effect of poly (I:C) transfection on HeLa cell apoptosis. (A) Effects of poly (I:C) transfection on HeLa cell apoptosis. The cells were stained with Annexin V/PI. (B) Quantification of the data shown in (A). (C) Western blot analysis of the protein levels of Bcl-2, Bax, Survivin and Bid. (D) Quantification of the data shown in $(\mathrm{C})$. Data are expressed as the mean \pm standard deviation. ${ }^{*}<0.05$ vs. the control group. poly (I:C), polyinosinic acid:polycytidylic acid; Bcl-2, B cell lymphoma-2-associated X protein; Bid, BH3 interacting-domain death agonist; PI, propidium iodide; FITC, fluorescein isothiocyanate.

dark. The cells were then washed twice in PBS and analyzed using flow cytometry (Cytomics FC 500; Beckman Coulter, Brea, CA, USA) or observed using a fluorescence microscope (BX53; Olympus Corporation, Tokyo, Japan). The redox state of the samples can be monitored by detecting increases in fluorescence. Accumulation of DCF in the cells is measured by an increase in fluorescence at $530 \mathrm{~nm}$.

Mitochondrial membrane potential $(\Delta \Psi m)$ assay. The mitochondrial $\Delta \Psi \mathrm{m}$ in the cells was determined according to a previous report (24). The HeLa cells were collected following the different treatments in 6-well plates. Following being washed twice with PBS, the cells were incubated with MitoProbe $^{\mathrm{TM}}$ 3, 3'-diethyloxacarbicyanine iodide using a Molecular Probes DiOC 2 (3) kit (Thermo Fisher Scientific, Inc.) at a concentration of $8 \mathrm{nM}$ for $30 \mathrm{~min}$ at $37^{\circ} \mathrm{C}$. These stained cells were examined using a flow cytometer (FACSCalibur; BD Biosciences).

Caspase activity assay. The cell lysates were prepared following the different treatments using cell lysis buffer (Beyotime Institute of Biotechnology). Briefly, $5 \times 10^{6}$ cells were suspended in $50 \mu \mathrm{l}$ chilled cell lysis buffer $(20 \mathrm{mM}$ Tris-HCl, pH 7.5; $150 \mathrm{mM} \mathrm{NaCl} ; 1 \mathrm{mM} \mathrm{Na} 2$ EDTA; $1 \mathrm{mM}$ EGTA; $1 \%$ Triton; $2.5 \mathrm{mM}$ sodium pyrophosphate; $1 \mathrm{mM}$ beta-glycerophosphate; $1 \mathrm{mM} \mathrm{Na} \mathrm{VO}_{4} ; 1 \mu \mathrm{g} / \mathrm{ml}$ leupeptin) and incubated on ice for $10 \mathrm{~min}$. Following centrifugation at $14,000 \mathrm{x} \mathrm{g}$ and $4^{\circ} \mathrm{C}$ for $10 \mathrm{~min}$, the supernatant (cytosolic extract) was transferred to a fresh tube and placed on ice, and $300 \mu \mathrm{g}$ of the protein was diluted in $50 \mu \mathrm{l}$ cell lysis buffer. The activity of caspase- 3 was determined using a Caspase-3 Activity kit (cat. no. C1116; Beyotime Institute of Biotechnology) according to the manufacturer's protocol. The assays were performed on 96-well microtitre plates by incubating $10 \mu \mathrm{l}$ cell lysate protein/sample in $80 \mu \mathrm{l}$ reaction buffer, containing 1\% NP-40, $20 \mathrm{mM}$ Tris- $\mathrm{HCl}(\mathrm{pH} 7.5)$, $137 \mathrm{mM} \mathrm{Nad}$ and $10 \%$ glycerol, and $10 \mu \mathrm{l}$ caspase-3 substrate (acetyl-Asp-Glu-Val-Asp p-nitroanilide; Ac-DEVDpNA; $2 \mathrm{mM}$; BioVision, Inc., Milpitas, CA, USA). The lysates were incubated at $37^{\circ} \mathrm{C}$ for $4 \mathrm{~h}$, following which the samples were measured using an ELISA reader (Labsystems, Helsinki, Finland) at an absorbance of $405 \mathrm{~nm}$ (25). Caspase-4 activity was measured using a commercially available Caspase-4 Assay kit (cat. no. C1122; Beyotime Institute of Biotechnology). The procedure was performed, according to the manufacturer's protocol. Briefly, $\sim 300 \mu \mathrm{g}$ protein was diluted in $50 \mu \mathrm{l}$ cell lysis buffer. Subsequently, $50 \mu 1$ of $2 \mathrm{X}$ reaction buffer, containing $10 \mathrm{mM}$ dithiothreitol, was added to each sample. Finally, $5 \mu \mathrm{l}$ of the $4 \mathrm{mM}$ LEVD-pNA substrate (final concentration, $200 \mu \mathrm{M}$; BioVision, Inc.) was added and incubated at $37^{\circ} \mathrm{C}$ for $1.5 \mathrm{~h}$. The absorbance was measured in an ELISA reader (Labsystems, Helsinki, Finland) at $405 \mathrm{~nm}$. The caspase-9 activity assay was performed using the caspase-9 Assay kit (cat. no. ab119508; Abcam), according to the manufacture's protocol, and the samples were prepared using the same method used for caspase-3, described above. Subsequently, $85 \mu 1$ reaction buffer and $5 \mu 1$ Leu-Glu-His-Asp-p-nitroanilide (LEHD-pNA) were added to each sample, and incubated at $37^{\circ} \mathrm{C}$ for $2 \mathrm{~h}$. The absorbance was measured in an ELISA reader (Labsystems, Helsinki, Finland) at $405 \mathrm{~nm}$.

Statistical analysis. Data are presented as the mean \pm standard deviation. The results were analyzed using a two-tailed t-test or one-way analysis of variance followed by Duncan's test to evaluate the differences among groups. Statistical analysis was performed using SPSS 13.0 (SPSS, Inc., Chicago, IL, USA). $\mathrm{P}<0.05$ was considered to indicate a statistically significant difference. 


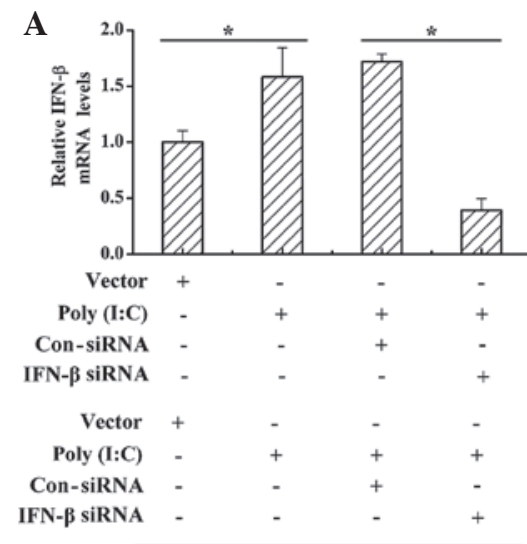

B
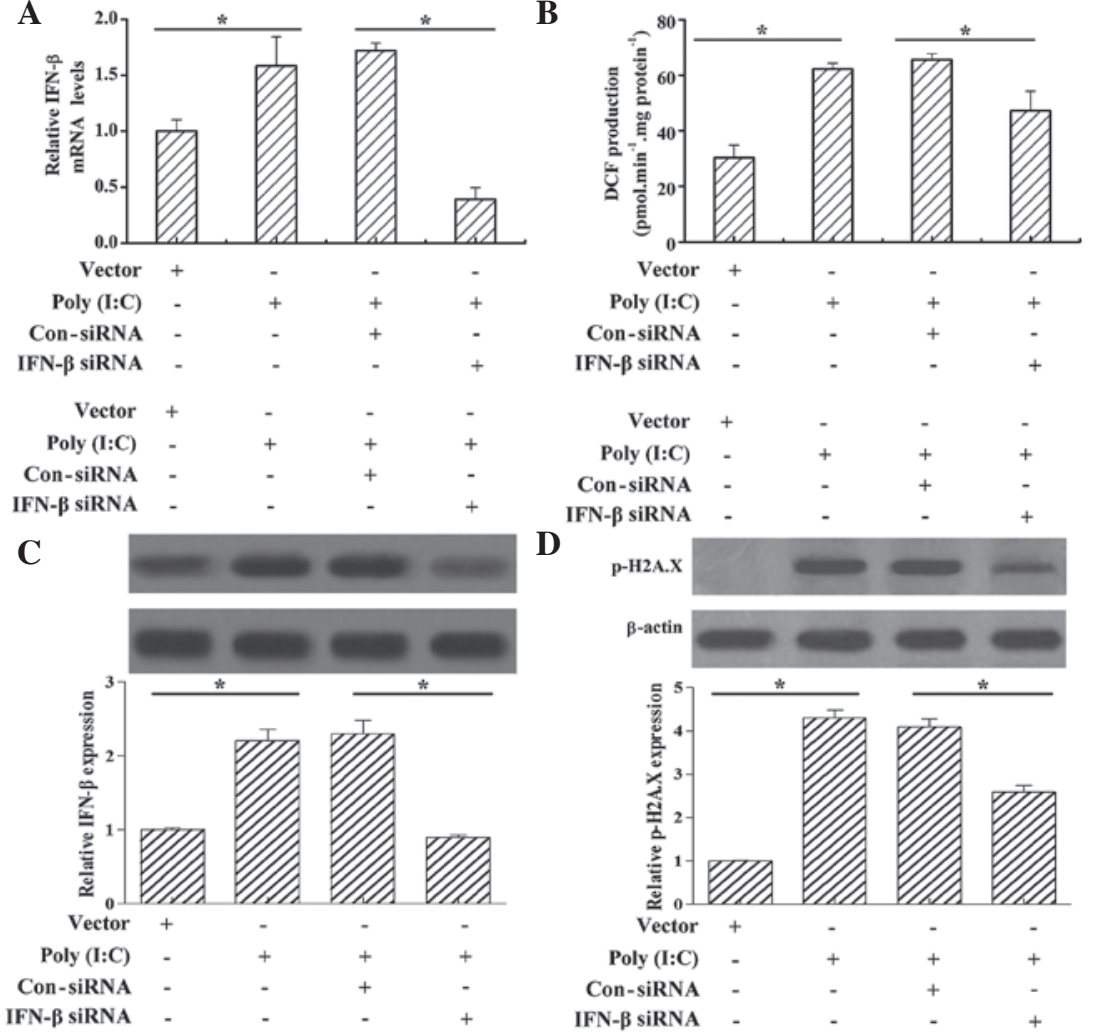

D

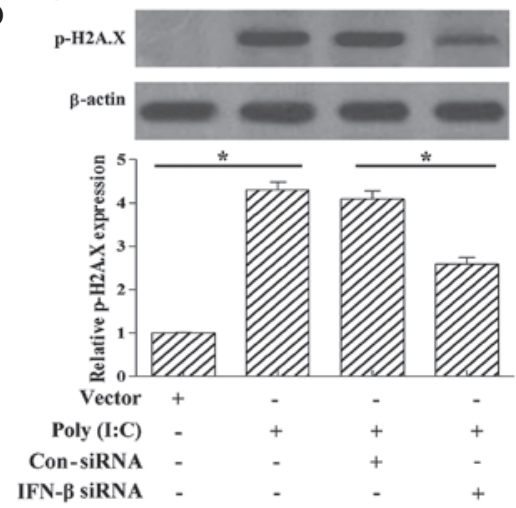

Figure 2. Effect of poly (I:C) transfection on the mRNA levels of IFN, production of ROS and DNA damage. (A) mRNA levels of IFN- $\beta$ in HeLa cells following poly (I:C) transfection. (B) ROS production following poly (I:C) transfection. (C) IFN- $\beta$ protein expression in HeLa cells following poly (I:C) transfection. (D) DNA damage, detected by analyzing the phosphorylation of $\gamma \mathrm{H} 2 \mathrm{~A} . \mathrm{X}$ at Ser 139 post-poly (I:C) transfection. Data are expressed as the mean \pm standard deviation. "P<0.05. poly (I:C), polyinosinic acid:polycytidylic acid; IFN, interferon; ROS, reactive oxygen species; DCF, dichlorofluorescein; p- $\gamma$ H2A.X; phosphorylated- $\gamma$ H2A.X; siRNA, small interfering RNA; Con, control.
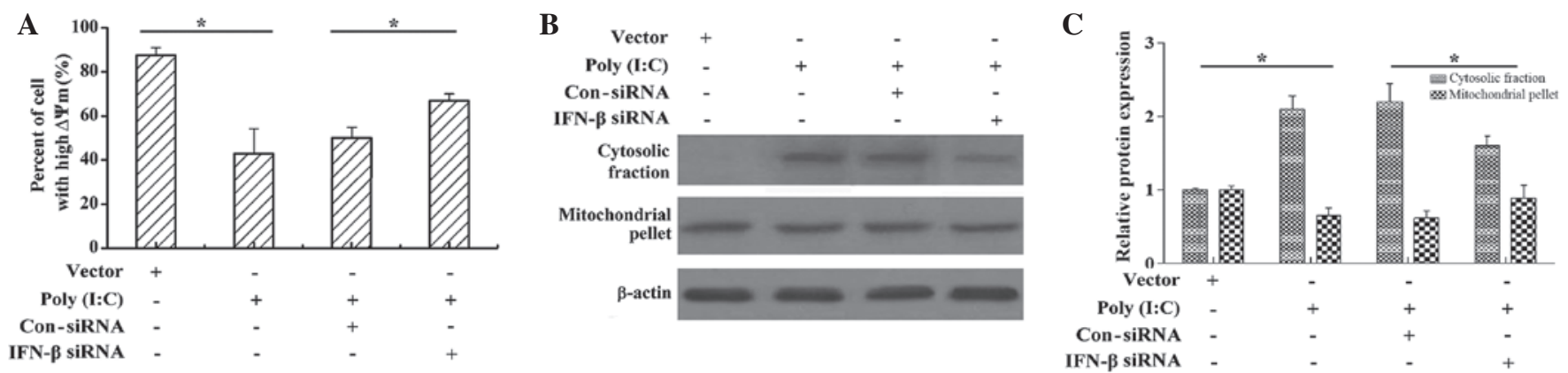

Figure 3. Effect of poly (I:C) transfection and IFN- $\beta$ knockdown on $\Delta \Psi \mathrm{m}$ and the release of cytochrome $c$ from the mitochondria to the cytoplasm. (A) $\Delta \Psi \mathrm{m}$ in the HeLa cells following poly (I:C) transfection and IFN- $\beta$ knockdown. (B) Content of cytochrome $c$ in the HeLa cells following poly (I:C) transfection and IFN- $\beta$ knockdown. (C) Quantification of band intensity of cytochrome $c$ in cytosolic fraction and mitochondrial pellet. Data are expressed as the mean \pm standard deviation. ${ }^{*} \mathrm{P}<0.05$. poly (I:C), polyinosinic acid:polycytidylic acid; IFN, interferon; $\Delta \Psi \mathrm{m}$, mitochondrial membrane potential; siRNA, small interfering RNA; Con, control.

\section{Results}

Poly (I:C) transfection induces cervical cancer cell apoptosis. The effects of poly (I:C) transfected into the HeLa cervical cancer cell line was first examined. Flow cytometry following Annexin V/PI staining revealed that poly (I:C) transfection increased the percentage of apoptosis cells between 4.5 and $65 \%$, compared with those in the control group (Fig. 1A and B). In addition, poly (I:C) transfection markedly increased the protein levels of the pro-apoptotic Bax and Bid, whereas it decreased the protein levels of anti-apoptotic Bcl-2 and
Survivin, compared with the control group. However, vector transfection had no marked effect on either cervical cancer cell apoptosis or the protein levels of the apoptosis-associated markers, Bacl-2, Bax, Survivin or Bid (Fig. 1C and D).

Poly (I:C) transfection increases the mRNA and protein levels of IFN- $\beta$, the production of ROS and DNA damage in cervical cancer cells. IFN- $\beta$ has been reported to be involved in apoptosis in cancer (26). In order to elucidate whether IFN- $\beta$ is involved in poly (I:C) transfection-induced cervical cancer cell apoptosis, the present study determined the mRNA and protein 


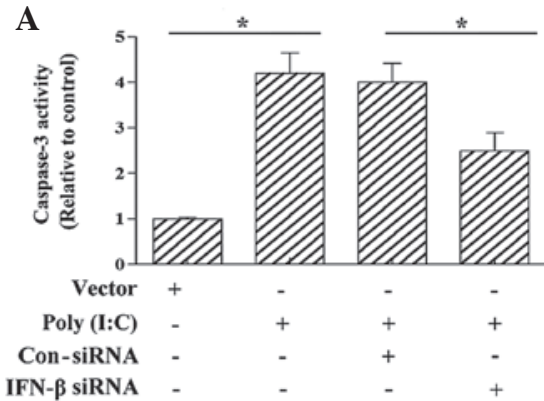

D

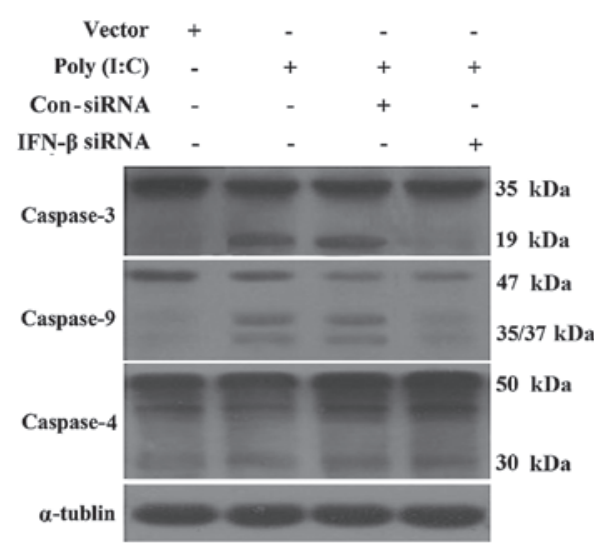

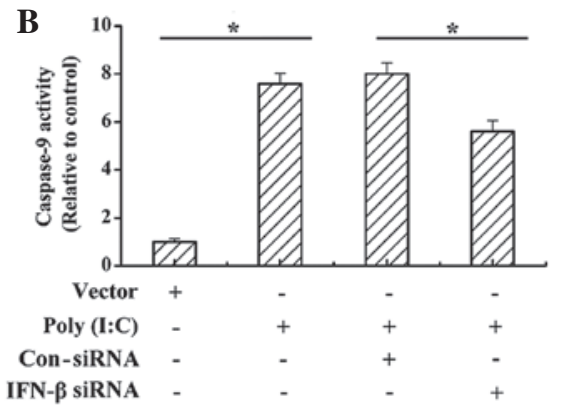

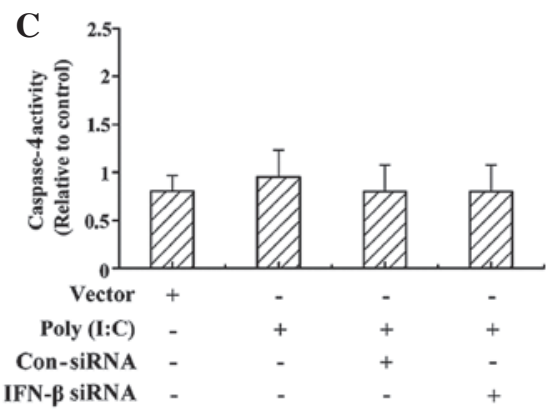

$\mathbf{E}$

Figure 4. Effect of poly (I:C) transfection and IFN- $\beta$ knockdown on caspase activation. (A) Caspase-3, (B) caspase-9 and (C) caspase-4 activity in the HeLa cells following poly (I:C) transfection and IFN- $\beta$ knockdown. (D) Western blot analysis of caspase- $9,-3,-4$ processing in the HeLa cells following poly (I:C) transfection and IFN- $\beta$ knockdown. (E) Quantification of caspase- $9,-3,-4$ protein band intensity. Data are expressed as the mean \pm standard deviation. "P<0.05. poly (I:C), polyinosinic acid:polycytidylic acid; IFN, interferon; siRNA, small interfering RNA; Con, control.

levels of IFN- $\beta$ following poly (I:C) transfection. It was found that vector transfection had no significant effect on the mRNA and protein levels of IFN- $\beta$; however, poly (I:C) transfection significantly promoted the mRNA and protein levels of IFN- $\beta$, compared with the vector control (Fig. 2A and C). An increase in ROS stress can induce apoptosis in cancer cells (27); in order to investigate whether poly (I:C) induced cancer cell apoptosis through the induction of oxidative stress, the present study determined whether poly (I:C) transfection triggered the generation of ROS. The results demonstrated that the staining intensity of carboxy-H2DCFDA increased significantly in the HeLa cells following poly (I:C) transfection, compared with the vector control, and this increase was inhibited by IFN- $\beta$ siRNA treatment (Fig. 2B). Excessive ROS production has the potential to damage cellular macromolecules, including DNA, eventually leading to cell death (28). In order to investigate whether poly (I:C) transfection also induced DNA damage, the present study examined DNA damage by analyzing the phosphorylation levels of $\gamma \mathrm{H} 2 \mathrm{~A}$.X at Ser 139. The western blot analyses showed that the levels of $\mathrm{p}-\gamma \mathrm{H} 2 \mathrm{~A}$.X increased in the HeLa cells following poly (I:C) transfection; however, treatment with IFN- $\beta$ siRNA subsequent to poly (I:C) transfection decreased the levels of p- $\gamma \mathrm{H} 2 \mathrm{~A} . \mathrm{X}$ (Fig. 2D).

Poly (I:C) transfection increases mitochondrial outer membrane permeabilization (MOMP) and cytochrome c release in cervical cancer cells. MOMP is often required for activation of the caspase proteases, which cause apoptotic cell death (29).

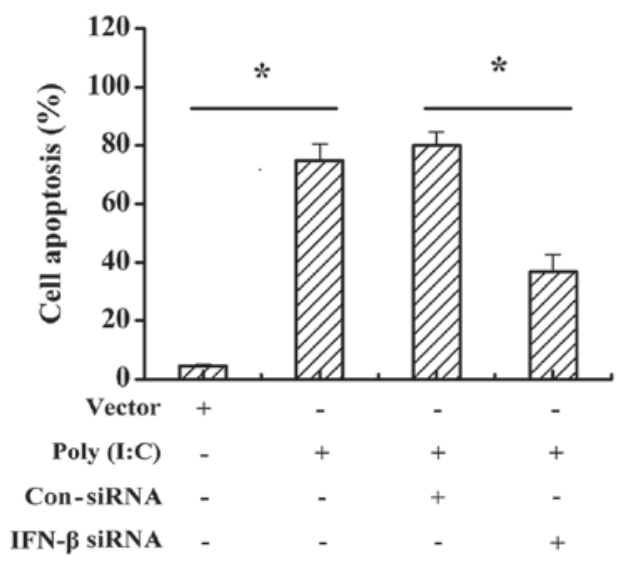

Figure 5. Effect of poly (I:C) transfection and IFN- $\beta$ knockdown on HeLa cell apoptosis. Data are expressed as the mean \pm standard deviation. ${ }^{*} \mathrm{P}<0.05$. poly (I:C), polyinosinic acid:polycytidylic acid; IFN, interferon; siRNA, small interfering RNA; Con, control.

To understand the underlying mechanism by which poly (I:C) transfection induces apoptosis, as well as the role of IFN- $\beta$ in this process, the present study investigated the change in $\Delta \Psi \mathrm{m}$, which is the cause of MOMP. Following poly (I:C) transfection, the number of cells exhibiting a high $\Delta \Psi \mathrm{m}$ decreased, compared with those transfected with the vector (Fig. 3A); and IFN- $\beta$ siRNA treatment following poly (I:C) transfection markedly increased the number of cells with a high $\Delta \Psi \mathrm{m}$. This data suggested that poly (I:C) transfection may disrupt the $\Delta \Psi \mathrm{m}$, 
and that IFN- $\beta$ is involved in this process (Fig. 3A). To further confirm the involvement of the mitochondrial signaling pathway during poly (I:C) transfection-induced apoptosis, the present study measured the release of cytochrome $c$ from the mitochondria into cytosol, a hallmark of mitochondria-mediated apoptosis. As shown in Figs. 3B and C, poly (I:C) transfection increased the content of cytosolic cytochrome $c$ and decreased the content of mitochondrial cytochrome $c$; however, IFN- $\beta$ siRNA treatment following poly (I:C) transfection decreased the content of cytosolic cytochrome $c$ and increased the content of mitochondrial cytochrome $c$. These results suggested that IFN- $\beta$ attenuated the poly (I:C)-induced release of cytochrome $c$ from mitochondria into cytosol.

Poly (I:C) transfection induces caspase-9 and caspase-3 activation. The release of cytochrome $c$ from the mitochondria into the cytosol is a key event for caspase activation (6). In order to further confirm whether poly (I:C) induced caspase activation, and whether IFN- $\beta$ was involved in the process of caspase activation, the present study subsequently examined caspase- 9 and caspase- 3 activity and processing. As shown in Fig. 4, poly (I:C) transfection significantly increased the activities of caspase- 3 and caspase- 9 , and IFN- $\beta$ siRNA markedly decreased the poly (I:C)-induced increases in caspase-3 and caspase-9 activity (Fig. 4A and B). The results of the western blot analysis showed that poly (I:C) transfection also promoted the cleavage of caspase-3 and caspase- 9 .

Caspase- 4 activation is required for endoplasmic reticulum (ER) stress-induced apoptosis in human cells (30). In order to investigate whether poly (I:C) transfection also induces cervical cancer cell apoptosis through the ER-mediated pathway, caspase-4 activity and processing were examined following poly (I:C) transfection. The results showed that poly (I:C) transfection marginally increased caspase-4 activity, however, the difference was not significant when compared with the vector-transfected group (Fig. 4C). In addition, IFN- $\beta$ siRNA had no significant effect on caspase- 4 activity (Fig. 4C). These results suggested that poly (I:C) induced the cervical cancer cell apoptosis predominantly through the mitochondrial-mediated pathway.

IFN- $\beta$ siRNA inhibits poly (I:C) transfection-induced cervical cancer cell apoptosis. The results described above suggested that poly (I:C) induced cervical cancer cell apoptosis, and that IFN- $\beta$ was involved in this progress. In order to confirm the involvement of IFN- $\beta$ in poly (I:C)-induced cervical cancer cell apoptosis, the present study determined the levels of cervical cancer cell apoptosis following poly (I:C) transfection and IFN- $\beta$ siRNA treatment. The results showed that poly (I:C) transfection significantly induced cervical cancer cell apoptosis, compared with vector transfection (Fig. 5). However, IFN- $\beta$ siRNA sharply attenuated the cervical cancer cell apoptosis induced by poly (I:C) transfection (Fig. 5). These results demonstrated that poly (I:C) induced cervical cancer cell apoptosis partly by promoting the expression of IFN- $\beta$.

\section{Discussion}

Poly (I:C) is an analogue of dsRNA, which has been demonstrated to be effective in antitumor immunotherapy $(31,32)$.
Poly (I:C) had been reported to suppress murine B16F10 melanoma growth (9) and induce apoptosis in human hepatocellular carcinoma (10). The results of the present demonstrated that poly (I:C) transfection induced apoptosis in the HeLa cervical cancer cell line. The Bcl-2 family of proteins consists of anti-apoptotic proteins, including Bcl-2 and Survivin, and pro-apoptotic molecules, including Bax and Bid (33-35). In the present study, it was also demonstrated that poly (I:C) transfection was associated with the upregulation of Bax and Bid, and downregulation of Bcl-2 and Survivin in the HeLa cells. These results indicated that poly (I:C) transfection induced cervical cancer cell apoptosis.

ROS accumulation has been shown to be important in mediating apoptosis, and DNA damage is considered to be the most common type of ROS-mediated damage (27). It has been reported that poly $(\mathrm{I}: \mathrm{C})$ transfection induces ROS-triggered apoptosis in human renal cell carcinoma (19). A similar observation was made in the present study, in which poly (I:C) transfection resulted in ROS production and DNA fragmentation, which may be contributing factors in apoptosis of the HeLa cells. Mitochondria are the major organelles for ROS production, and excessive ROS accumulation contributes to cell/tissue injury or death (36-38). In the intrinsic pathway, MOMP, which leads to the release of proapoptotic proteins from the mitochondrial intermembrane space, including cytochrome $c$, promote caspase activation following their release from the mitochondria into the cytosol (6). The present study confirmed that poly (I:C) transfection decreased $\Delta \Psi \mathrm{m}$ in the HeLa cells, and induced the release of cytochrome $c$ from the mitochondria into the cytosol. The release of cytochrome $c$ induces an initiator caspase, for example caspase-9 activation, which subsequently triggers the cleavage and activation of caspase-3 and caspase-7 (6). The present study also demonstrated that poly ( $\mathrm{I}: \mathrm{C})$ transfection induced caspase- 9 and caspase- 3 activation in the HeLa cells. To elucidate whether poly (I:C) transfection induced apoptosis via the ER stress-mediate apoptosis pathway, caspase- 4 activity and processing were examined, with the results demonstrating that poly (I:C) transfection induced a marginal effect on caspase-4 activation. Together, these results suggested that the apoptosis of cervical cancer cells induced by poly (I:C) was predominantly triggered via the mitochondrial apoptotic pathway.

IFNs are a family of natural glycoproteins, which consist of IFN- $\alpha$, IFN- $\beta$ and IFN- $\gamma$ (17), and IFN- $\beta$ has been reported to be induced apoptosis in melanoma cell lines (26). In the present study, it was demonstrated that poly (I:C) transfection induced the mRNA expression of IFN- $\beta$ in the HeLa cells. In addition, IFN- $\beta$ knockdown significantly attenuated poly (I:C) transfection-induced ROS production, DNA damage, MOMP and cytochrome $c$ release, as well as caspase- 9 and caspase- 3 activation, in the HeLa cells. These results suggested that IFN- $\beta$ is likely to be involved in poly (I:C)-induced aoptosis in HeLa cells. The results of the present study also demonstrated that IFN- $\beta$ knockdown significantly restored poly (I:C) transfection-induced apoptosis in the HeLa cells. This result confirmed that poly (I:C) transfection induced HeLa apoptosis through the IFN- $\beta$ signaling pathway.

In conclusion, the results of the present study indicated that poly (I:C) contributed to the apoptosis of cervical cancer cells via the mitochondrial apoptotic pathway. In addition, poly (I:C) 
transfection regulated cervical cancer cell apoptosis through the IFN- $\beta$ signaling and the intrinsic mitochondrial apoptotic pathway. However, the exact regulatory mechanism of poly (I:C) and IFN- $\beta$ in cervical cancer cells requires further clarification. These findings indicate that poly (I:C) may be considered a competitive candidate for the treatment of cervical cancer.

\section{References}

1. Einstein MH, Schiller JT, Viscidi RP, Strickler HD, Coursaget P, Tan T, Halsey N and Jenkins D: Clinician's guide to human papillomavirus immunology: Knowns and unknowns. Lancet Infect Dis 9: 347-356, 2009.

2. Chauhan SC, Jaggi M, Bell MC, Verma M and Kumar D: Epidemiology of human papilloma virus (hpv) in cervical mucosa. Methods Mol Biol 471: 439-456, 2009.

3. Ashkenazi A and Dixit VM: Death receptors: signaling and modulation. Science. 281: 1305-1308, 1998.

4. Li P, Nijhawan D, Budihardjo I, Srinivasula SM, Ahmad M, Alnemri ES and Wang X: Cytochrome c and dATP-dependent formation of Apaf-1/caspase-9 complex initiates an apoptotic protease cascade. Cell 91: 479-489, 1997.

5. Ma Y, Zhang J, Zhang Q, Chen P, Song J, Yu S, Liu H, Liu F, Song C, Yang D and Liu J: Adenosine induces apoptosis in human liver cancer cells through ROS production and mitochondrial dysfunction. Biochem Biophys Res Commun 448: 8-14, 2014

6. Tait SW and Green DR: Mitochondria and cell death: Outer membrane permeabilization and beyond. Nat Rev Mol Cell Bio 11: 621-632, 2010.

7. Li H, Zhu H, Xu CJ and Yuan J: Cleavage of BID by caspase 8 mediates the mitochondrial damage in the Fas pathway of apoptosis. Cell 94: 491-501, 1998.

8. Singh KK: Mitochondria damage checkpoint, aging and cancer. Ann NY Acad Sci 1067: 182-190, 2006

9. Fujimura T, Nakagawa S, Ohtani T, Ito Y and Aiba S: Inhibitory effect of the polyinosinic-polycytidylic acid/cationic liposome on the progression of murine B16F10 melanoma. Eur J Immunol 36 : 3371-3380, 2006.

10. Zhang Y, Lin A, Sui Q, Zhang C, Tian Z and Zhang J: Phosphorothioate modification of the TLR9 ligand CpG ODN inhibits poly (I:C)-induced apoptosis of hepatocellular carcinoma by entry blockade. Cancer Lett 355: 76-84, 2014.

11. Peng S, Geng J, Sun R, Tian Z and Wei H: Polyinosinic-pol ycytidylic acid liposome induces human hepatoma cells apoptosis which correlates to the up-regulation of RIG-I like receptors. Cancer Sci 100: 529-536, 2009.

12. Sottini A, Capra R, Serana F, Chiarini M, Caimi L and Imberti L: Interferon-beta therapy monitoring in multiple sclerosis patients. Endocr Metab Immune Disord Drug Targets 9: 14-28, 2009.

13. Cha L, de Jong E, French MA and Fernandez S: IFN- $\alpha$ exerts opposing effects on activation-induced and IL-17-induced proliferation of $\mathrm{T}$ cells that may impair homeostatic maintenance of $\mathrm{CD}^{+} \mathrm{T}$ cell numbers in treated HIV infection. J Immunol 193 2178-2186, 2014.

14. Hong B, Li H, Lu Y, Zhang M, Zheng Y, Qian J and Yi Q: USP18 is crucial for IFN- $\gamma$-mediated inhibition of B16 melanoma tumorigenesis and antitumor immunity. Mol Cancer 13: 132, 2014.

15. Ryu H, Oh JE, Rhee KJ, Baik SK, Kim J, Kang SJ, Sohn JH, Choi E, Shin HC and Kim YM: Adipose tissue-derived mesenchymal stem cells cultured at high density express IFN- $\beta$ and suppress the growth of MCF-7 human breast cancer cells. Cancer Lett 352: 220-227, 2014

16. Matsuzuka T, Miller K, Pickel L, Doi C, Ayuzawa R and Tamura M: The synergistic induction of cyclooxygenase- 2 in lung fibroblasts by angiotensin II and pro-inflammatory cytokines. Mol Cell Biochem 320: 163-171, 2009.

17. Takano S, Ishikawa E, Matsuda M, Yamamoto T and Matsumura A: Interferon- $\beta$ inhibits glioma angiogenesis through downregulation of vascular endothelial growth factor and upregulation of interferon inducible protein 10. Int J Oncol 45: 1837-1846, 2014.
18. Jablonska J, Leschner S, Westphal K, Lienenklaus S and Weiss S: Neutrophils responsive to endogenous IFN-beta regulate tumor angiogenesis and growth in a mouse tumor model. J Clin Invest 120: 1151-1164, 2010

19. Harashima N, Minami T, Uemura H and Harada M: Transfection of poly (I:C) can induce reactive oxygen species-triggered apoptosis and interferon- $\beta$-mediated growth arrest in human renal cell carcinoma cells via innate adjuvant receptors and the 2-5A system. Mol Cancer 13: 217, 2014.

20. Siegal FP, Kadowaki N, Shodell M, Fitzgerald-Bocarsly PA, Shah K, Ho S, Antonenko S and Liu YJ: The nature of the principal type 1 interferon-producing cells in human blood. Science 284: 1835-1837, 1999.

21. Livak KJ and SchmittgenTD: Analysis of relative gene expression data using real-time quantitative PCR and the 2(-Delta Delta C(T)) Method. Methods 25: 402-408, 2001.

22. Laggner H, Hermann M, Gmeiner BM and Kapiotis $\mathrm{S}: \mathrm{Cu}^{2+}$ and $\mathrm{Cu}^{+}$bathocuproine disulfonate complexes promote the oxidation of the ROS-detecting compound dichlorofluorescin (DCFH). Anal Biochem Chem 385: 959-961, 2006

23. Yim HY, Yang Y, Lim JS, Lee MS, Zhang DE and Kim KI: The mitochondrial pathway and reactive oxygen species are critical contributors to interferon- $\alpha / \beta$-mediated apoptosis in Ubp43-deficient hematopoietic cells. Biochem Biophys Res Commun 423: 436-440, 2012

24. Rottenberg $\mathrm{H}$ and $\mathrm{Wu} \mathrm{S}$ : Quantitative assay by flow cytometry of the mitochondrial membrane potential in intact cells. Biochim Biophys Acta 1404: 393-404, 1998.

25. Liu Y, Zhang SP and Cai YQ: Cytoprotective effects of selenium on cadmium-induced LLC-PK1 cells apoptosis by activating JNK pathway. Toxicol In Vitro 21: 677-684, 2007.

26. Chawla-Sarkar M, Leaman DW and Borden EC: Preferential induction of apoptosis by interferon (IFN)-beta compared with IFN-alpha2: Correlation with TRAIL/Apo2 L induction in melanoma cell lines. Clin Cancer Res 7: 1821-1831, 2001.

27. Pelicano H, Carney D and Huang P: ROS stress in cancer cells and therapeutic implications. Drug Resist Update 7: 97-110, 2004.

28. Hensley K, Robinson KA, Gabbita SP, Salsman S and Floyd RA: Reactive oxygen species, cell signaling and cell injury. Free Radical Bio Med 28: 1456-1462, 2000

29. Gillies LA and Kuwana T: Apoptosis regulation at the mitochondrial outer membrane. J Cell Biochem 115: 632-640, 2014.

30. Li C, Wei J, Li Y, He X, Zhou Q, Yan J, Zhang J, Liu Y, Liu Y and Shu HB: Transmembrane Protein 214 (TMEM214) mediates endoplasmic reticulum stress-induced caspase 4 enzyme activation and apoptosis. J Biol Chem 288: 17908-17917, 2013.

31. Pimm MV and Baldwin RW: Treatment of transplanted rat tumours with double-stranded RNA (BRL 5907). II. Treatment of pleural and peritoneal growths. Br J Cancer 33: 166-171, 1976.

32. Pimm MV, Embleton MJ and Baldwin RW: Treatment of transplanted rat tumours with double-stranded RNA (BRL 5907). I. Influenced of systemic and local administration. Br J Cancer 33: 154-165, 1976.

33. Adams JM and Cory S: The Bcl-2 apoptotic switch in cancer development and therapy. Oncogene 26: 1324-1337, 2007.

34. Kang BH, Xia F, Pop R, Dohi T, Socolovsky M and Altieri DC: Developmental control of apoptosis by the immunophilin aryl hydrocarbon receptor-interacting protein (aip) involves mitochondrial import of the survivin protein. J Biol Chem 286: 16758-16767, 2011.

35. Croker BA, O'Donnell JA, Nowell CJ, Metcalf D, Dewson G, Campbell KJ, Rogers KL, Hu Y, Smyth GK, Zhang JG, et al: Fas-mediated neutrophil apoptosis is accelerated by Bid, Bak and Bax and inhibited by Bcl-2 and Mcl-1. Proc Natl Acad Sci USA 108: 13135-13140, 2011.

36. Kang J and Pervaiz S: Mitochondria: Redox metabolism and dysfunction. Biochem Res Int 2012: 896751, 2012.

37. Yoboue ED and Devin A: Reactive oxygen species-mediated control of mitochondrial biogenesis. Int J Cell Biol 2012: 403870 , 2012.

38. Yadav N and Chandra D: Mitochondrial and postmitochondrial survival signaling in cancer. Mitochondrion 16: 18-25, 2014. 